\title{
Seasonal variation of turbulent energy dissipation rates in the polar mesosphere: a comparison of methods
}

\author{
C. M. Hall ${ }^{1}$, U.-P. Hoppe ${ }^{2}$, T. A. Blix², E. V. Thrane², A. H. Manson ${ }^{3}$, and C. E. Meek ${ }^{3}$ \\ ${ }^{1}$ Department of Physics, University of Troms $\phi, 9037$ Troms $\phi$, Norway \\ ${ }^{2}$ Norwegian Defence Research Establishment, 2027 Kjeller, Norway \\ ${ }^{3}$ Institute of Space and Atmospheric Studies, University of Saskatchewan, Saskatoon, Saskatchewan, Canada
}

(Received June 29, 1998; Revised January 20, 1999; Accepted June 3, 1999)

\begin{abstract}
During the last two decades many estimates of turbulence strength have been made by a variety of techniques in the mesosphere above northern Norway. We have assimilated many of these results and present them in this study, enabling the reader to note systematic differences. We concentrate on seasonal variation not only in an attempt to smooth out non-representative data, but also to identify the seasonal features themselves. We note both semi-annual and annual variations in turbulent intensity, depending on the height considered. Finally we address the aforementioned systematic differences between the methods and suggest possible causes in terms of each method's underlying assumptions.
\end{abstract}

\section{Introduction}

It is no coincidence that a broad spectrum of ground-based instrumentation has been built up in the vicinities of Andenes and Troms $\varnothing$ in Northern Scandinavia and furthermore in the vicinity of a sounding rocket launching facility (Fig. 1). The available instrumentation has provided the scientific community with both ground-based and in situ measurements of the mesosphere over a period of almost 2 decades and it is now becoming possible to study such data in a statistical way. Results from ion-, electron- and neutral density probes, EISCAT (European Incoherent Scatter Radar) and the University of Troms $\varnothing /$ University of Saskatchewan MF radar system have been selected for this study. We shall address the turbulent energy dissipation rate, $\epsilon$, in this study. $\epsilon$ is a commonly derived entity for parameterising turbulent intensity and is easily converted to a heating rate in $\mathrm{K} / \mathrm{day}$, which is a conveniently understandable unit. We have selected 4 basic methods of estimating $\epsilon$, the results from which we will present and intercompare. We shall find agreement and disagreement between the methods and for the latter case briefly discuss the possible reasons. A paper by Hocking (1999) in this issue represents a critique of much of the theory underlying these derivations. For this reason we include commonlyused formulae in this paper to facilitate reference to Hocking (1999). Clearly, to identify why one well-established method yields significantly different results from another is crucial to future research and not least, financial investment in new instruments and observations. Furthermore, different methods have advantages and disadvantages compared to others. A secondary object of this study is to help identify which kind of observation is best suited to which kind of study. For

Copy right (C) The Society of Geomagnetism and Earth, Planetary and Space Sciences (SGEPSS); The Seismological Society of Japan; The Volcanological Society of Japan; The Geodetic Society of Japan; The Japanese Society for Planetary Sciences. example, high spatial-resolution case-study kinds of measurements are best performed in situ; while studies of diurnal variations would be expensive using rockets, ground-based experiments are perhaps better suited for this purpose. MF radar systems are relatively cheap to build but often suffer from poor height resolution. In addition, they are often affected by group delay of the radio wave by the ionosphere, and so the exact heights of the observations can be uncertain above $90 \mathrm{~km}$. Perhaps an even more important contaminant is the leading edge of any very large E-region echo "leaking" into the MF data at $\sim 92 \mathrm{~km}$ and above (Hocking, 1997). The methods we shall describe in more detail below are tabulated along with the main advantages and disadvantages in Table 1. We should stress that the table is indicating which method is best suited to a particular kind of observation, not that one or more methods should be discarded altogether. Furthermore, it is important to note that while some methods attempt to estimate the turbulent energy dissipation rate itself, others rather determine an upper limit for it. Differences between the "upper-limit" estimates and the actual energy dissipation rate characterising the inertial subrange of turbulence arise from processes that "violate" the classical energy cascade. Also, we shall see that many methods employ a length scale, often an estimated "outer scale" (or largest scale enjoyed by inertial processes); buoyant dynamics smaller in scale than this estimate will also cause derived dissipation rates to be larger than the purely turbulent energy disspation rate.

Throughout the forthcoming descriptions of the various methods, we need to employ values for the Brunt-Väisälä frequency; we normally take this from the MSISE90 (Hedin, 1991) model temperatures which offer good continuity in both time and height. Where previously published data has been included, other models and/or measurements may have been used and these are either quoted in the text or available via the corresponding reference. 


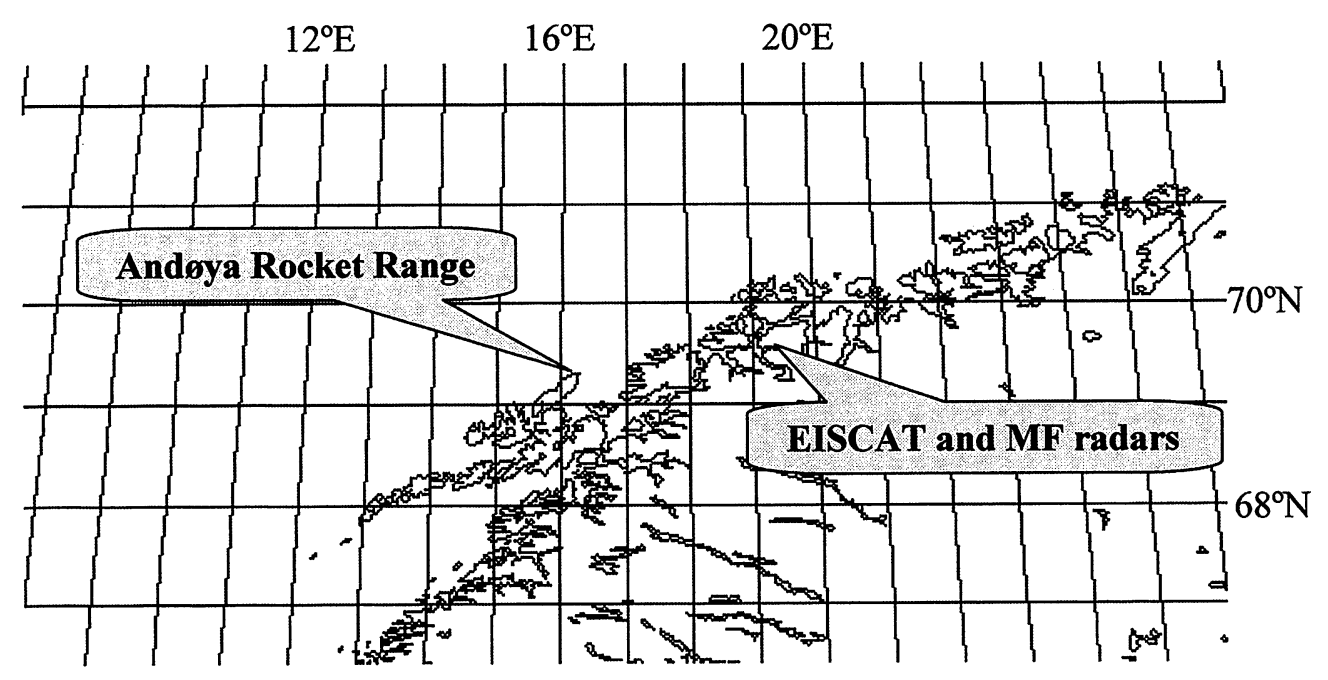

Fig. 1. Map of Scandinavia indicating the locations of the instruments listed in Table 1.

Table 1. Overview of instrumentation for determining mesospheric turbulence parameters above the Troms $\varnothing /$ Andøya region of Northern Norway.

\begin{tabular}{lll}
\hline Method & pro & contra \\
\hline In situ & Very high resolution & Expensive for longer-term coverage \\
MF radar fading time & Continuous monitoring cheap & Poor height resolution \\
EISCAT vertical wavenumber & Continual monitoring & Low spatial resolution \\
EISCAT velocity fluctuation & Continual monitoring & Too low spatial resolution \\
\hline
\end{tabular}

\section{In Situ Methods}

It is not trivial to determine turbulence-induced fluctuations in density of the neutral atmosphere. A detector with a sufficiently high sampling rate to determine fluctuations at scales as small as the viscous subrange of turbulence is difficult to construct, especially so if it is to be integrated into a rocket payload. One method is to assume that the ionised components of the atmosphere can be used as passive scalar tracers for the neutral gas; thus the density fluctuations of ions and/or electrons are determined and neutral density fluctuations are deduced (e.g., Blix et al., 1990). An alternative is to ionise the neutral particles in situ and measure the resulting ion density fluctuations (e.g., Hillert et al., 1993). Yet another method is to attempt to measure the neutral density fluctuations directly, but it is only recently that suitable miniaturisation of the necessary technology has been possible (Hoppe et al., (1999) this issue). All methods ultimately attempt to determine neutral velocity fluctuations as functions of either time or space, and at sufficient resolution to investigate turbulent scales, since $\epsilon$ is a measure of the way kinetic energy is dissipated into heat by viscosity. Often it is neutral density fluctuations that are determined and therefore these density fluctuations must be used as the basis for kinetic energy fluctuations. In early studies equipartition of energy between potential and kinetic was assumed, but recently this has been seen as an oversimplification (e.g., Hocking, 1992, 1996). The spectra of the kinetic energy fluctuations often agree with the theoretical form predicted by Kolmogorov (1941) and further modelled by Heisenberg (1948) and Tartarskii (1971). Either the whole form of the spectrum may be utilised to estimate the inner scale (Lübken, 1996), or the power spectral density in the inertial subrange can be estimated (e.g., Blix et al., 1990).

All soundings included here are summarised in Table 2, and Table 3 indicates the numbers of soundings for each month. We shall examine the various methods below, all of which attempt to determine $\epsilon$ in its true sense, as opposed to some upper limit. For information on errors, instrumental and otherwise, the reader is referred to the references given in the sections that follow. The uncertainties incurred in using models and the various constants involved in estimation of turbulence strength will usually far outweigh instrumental noise.

\subsection{Positive ion probes (PIP)}

Two types of probe, spheres and half-spheres, have been flown. Each consists of an outer grid at rocket potential and an inner collector (solid or grid) biased $-7 \mathrm{~V}$ with respect to the outer grid. The measured current is due to positive ions and was measured with a resolution of 12 bits (in addition 3 bits were used for range indication) and a sampling frequency in the range $2000-2500 \mathrm{~Hz}$. Taking the rocket velocity into account and assuming no temporal instrumental smearing, this corresponds to a spatial resolution of better than $0.5 \mathrm{~m}$. The probes measured currents in the range $10^{-11}$ to $10^{-4} \mathrm{~A}$, varying slightly from campaign to campaign. The probes were therefore able to measure very small-scale fluctuations 
Table 2. Details of rocket soundings used in this paper. Further details can be found via the references given in the text along with the designation given in the first column.

\begin{tabular}{|c|c|c|}
\hline Rocket designation & Launch date and time & \\
\hline F-52 PIP & 11 Nov. 1980 03:24 & 1 \\
\hline F-53 PIP & 16 Nov. 1980 03:31 & 1 \\
\hline F-54 PIP & 11 Nov. 1980 00:12 & 1 \\
\hline F-55 PIP & 16 Nov. 1980 03:31 & 1 \\
\hline F-56 PIP & 28 Nov. 1980 03:24 & 1 \\
\hline F-56 PIP & 28 Nov. 1980 03:24 & 1 \\
\hline F-56 PIP & 28 Nov. 1980 03:24 & 1 \\
\hline F-56 PIP & 28 Nov. 1980 03:24 & 1 \\
\hline F-57 PIP & 11 Nov. 1980 00:12 & 1 \\
\hline F-57 PIP & 11 Nov. 1980 00:12 & 1 \\
\hline F-64 PIP & 16 Feb. 1984 01:20 & 2 \\
\hline F-65 PIP & 18 Feb. 1984 00:22 & 2 \\
\hline F-66 PIP & 6 Jan. 1984 21:55 & 2 \\
\hline F-67 PIP & 25 Jan. 1984 17:39 & 2 \\
\hline F-68 PIP & 13 Jan. 1984 20:00 & 2 \\
\hline F-69 PIP & 10 Feb. 1984 02:40 & 2 \\
\hline F-70 PIP & 31 Jan. 1984 18:31 & 2 \\
\hline F-74 PIP & 12 Nov. 1987 00:16 & 3 \\
\hline F-75 PIP & 21 Oct. 1987 21:33 & 3 \\
\hline F-76 PIP & 15 Oct. 1987 10:52 & 3 \\
\hline F-77 PIP & 21 Oct. 1987 21:33 & 3 \\
\hline F-78 PIP & 12 Nov. 1987 00:21 & 3 \\
\hline F-81 PIP and TOTAL & 22 Jan. 1990 10:20 & 4 \\
\hline F-82 PIP and TOTAL & 25 Feb. 1990 19:20 & 5 \\
\hline F-83 PIP and TOTAL & 6 Oct. 1990 02:41 & 5 \\
\hline F-84 PIP and TOTAL & 8 Mar. 1990 22:53 & 5 \\
\hline F-85 PIP and TOTAL & 9 Mar. 1990 00:25 & 5 \\
\hline F-86 PIP and TOTAL & 11 Mar. 1990 20:42 & 5 \\
\hline F-90 TOTAL & 1 Aug. 1991 01:40 & 6 \\
\hline F-91 PIP and TOTAL & 9 Aug. 1991 23:15 & 6 \\
\hline F-95 PIP and TOTAL & 17 Sept. 1991 23:43 & 7 \\
\hline F-96 PIP and TOTAL & 20 Sept. 1991 20:48 & 7 \\
\hline F-93 TOTAL & 20 Aug. 1991 22:40 & 7 \\
\hline F-97 PIP and TOTAL & 30 Sept. 1991 20:55 & 7 \\
\hline F-98 PIP and TOTAL & 3 Oct. 1991 22:27 & 7 \\
\hline F-99 PIP and TOTAL & 4 Oct. 1991 00:08 & 7 \\
\hline F-100 CONE & 28 Jul. 1993 22:23 & 8 \\
\hline F-94 PIP and CONE & 1 Aug. 1993 01:46 & 8 \\
\hline F-102 CONE & 28 Jul. 1993 22:39 & 9 \\
\hline F-103 CONE & 31 Jul. 1993 00:50 & 9 \\
\hline F-101 PIP and CONE & 1 Aug. 1993 00:53 & 9 \\
\hline Super Arcas 1 NTP & 14 Jul. 1987 08:00 & 10 \\
\hline Super Arcas 2 NTP & 14 Jul. 1987 09:29 & 10 \\
\hline Super Arcas 3 NTP & 14 Jul. 1987 12:55 & 10 \\
\hline Super Arcas 4 NTP & 15 Jul. 1987 12:32 & 10 \\
\hline
\end{tabular}

${ }^{1}$ Energy Budget Campaign, ${ }^{2} \mathrm{MAP} / \mathrm{WINE},{ }^{3} \mathrm{MAC} / \mathrm{EPSILON}$, ${ }^{4}$ Recommend, ${ }^{5}$ DYANA, ${ }^{6}$ NLC-91, ${ }^{7}$ METAL, ${ }^{8}$ SCALE, ${ }^{9}$ ECHO, ${ }^{10} \mathrm{MAC} / \mathrm{SINE}$.
Table 3. Numbers of soundings used to obtain each of the month averages described in the text.

\begin{tabular}{lc}
\hline Month & Number of soundings \\
\hline January & 7 \\
February & 6 \\
March & 11 \\
April & 0 \\
May & 0 \\
June & 0 \\
July & 7 \\
August & 6 \\
September & 9 \\
October & 9 \\
November & 13 \\
December & 0 \\
\hline
\end{tabular}

(down to $0.02 \%$ ) with high spatial resolution in the middle atmosphere.

We will, below, describe how we have treated the data from the different soundings performed during the years (19801994). The aim has been to treat all data in a consistent manner so that they can be directly compared and used as the basis for the annual variations discussed later. Early rocket sounding data analyses employed the US Standard atmosphere (NOAA-S/T76-1562, 1976), to obtain neutral atmosphere scale heights for pressure $\left(H_{\mathrm{p}}\right)$ and density $\left(H_{\mathrm{n}}\right)$, and we have used the same throughout for consistency. The scale height of the ion density $\left(H_{\mathrm{i}}\right)$ has been derived directly from the ion probe data. The significance of these parameters is obvious from the following discussion.

If the original telemetry data were available (soundings from 1990 onwards) we have performed the analysis described in detail by Thrane et al. (1985), again including any compensation for negative ions. For each sounding (upleg and/or downleg) we assembled one-kilometre altitude time series (cf. Blix et al.'s (1990) 1024 points). Having obtained sets of well behaved time series, a digital filter was applied giving a bandpass from $4 \mathrm{~Hz}$ (rejecting the spin frequency) to around $60 \mathrm{~Hz}$, corresponding to $15 \mathrm{~m}$. The variances of the results were then converted to neutral density fluctuations, $\Delta n / n$, according to the method of Blix et al. (1990). The corresponding potential energy (per unit mass) fluctuation $P E^{\prime}$ is derived from

$$
P E^{\prime}=z g \frac{\Delta n}{n}
$$

where $z$ is the vertical displacement of an air parcel. Assuming adiabatic vertical displacement of air parcels, Thrane and Grandal (1981) found the following relation between $\Delta n / n$ and $z$ :

$$
\frac{\Delta n}{n}=\left[\frac{1}{H_{\mathrm{n}}}-\frac{1}{\gamma H_{\mathrm{p}}}\right] z
$$

where $H_{\mathrm{n}}$ and $H_{\mathrm{p}}$ are the neutral density and pressure scale 
heights respectively. This expression is very similar to equation 17 of Hocking (1985) except that in this case scale heights are used whereas Hocking used gradients. The specific $\left(\mathrm{kg}^{-1}\right)$ kinetic energy fluctuation $K E^{\prime}=\frac{1}{2} U^{2}, U$ being the turbulent velocity. $U$ can be related to the horizontal $(u$ and $v$ ) and vertical $(w)$ components as $U^{2}=u^{2}+v^{2}+w^{2}=$ $C w^{2}$, where $C$ is constant describing the degree of isotropy. Thus, $K E^{\prime}=C \cdot \frac{1}{2} w^{2}$. The relation between $K E^{\prime}$ and $P E^{\prime}$ is given by the equation:

$$
K E^{\prime}=P E^{\prime}\left[\left(1-R_{\mathrm{i}}\right) / R_{\mathrm{i}}\right]
$$

(Weinstock, 1978; Hocking, 1992). We have taken $R_{\mathrm{i}}=$ 0.44 , this being suggested by (Weinstock, 1978) as a value representative of established and maintained turbulence. The relation (3) above is valid for $0<R_{\mathrm{i}} \leq 1$ (see Hocking (1992) for further details). It is unlikely, however, that one can characterise the atmosphere with any single value of $R_{\mathrm{i}}$; see Hocking and $\mathrm{Mu}$ (1997) for a more detailed discussion, including variations on Eq. (3) in terms of flux Richarson Number. The $w^{2}$ derivation of $\epsilon$ is then given by

$$
\epsilon=0.4 w^{2} \omega_{\mathrm{B}}=0.8 K E^{\prime} \omega_{\mathrm{B}} / C
$$

$\omega_{\mathrm{B}}$ being the Brunt-Väisälä frequency. We have assumed isotropic turbulence $(C=3)$ in our derivation of $\epsilon$. One must be careful about adopting the factor 3 however; there may be additional re-scaling constants of the order of $4 / 3$ depending on whether $w$ fluctuations are measured parallel or perpendicular to the motion of the detector through the turbulence.

If the original telemetry data were unavailable (prior to 1990), we have taken the published profiles of $\epsilon$ (all appropriate references can be found in Blix et al. (1990)), and converted them to profiles of neutral density fluctuation by exactly reversing the process described in detail by Blix et al. (1990). First, we obtain the structure function constant $C_{\mathrm{n}}^{2}$ using:

$$
C_{\mathrm{n}}^{2}=(\epsilon / 0.293)^{2 / 3} B\left[M / \omega_{\mathrm{B}}\right]^{2}
$$

where $M=\left(\gamma H_{\mathrm{p}} / H_{\mathrm{n}}-1\right) / \gamma H_{\mathrm{p}}$. Here, $B$ is a factor described by Blix et al. (1990) giving the relation between horizontal and vertical gradients. $B=3$ corresponds to the case that fluctuations at scales greater than $L_{B}$, the outer scale, are isotropic. $B=1$ corresponds to the case that scales greater than $L_{B}$ are stratified. Scales smaller than $L_{B}$ and in the inertial subrange are actually isotropic in both cases. For consistency with other results we shall incorporate, we have chosen to use $B=3$ in accordance with Blix et al. (1990) in our derivation. Then we obtain the power spectral density $P(f)$ at frequency $f_{0}$ from:

$$
C_{\mathrm{n}}^{2}=8 P_{n}(f)\left(2 \pi / V_{R}\right)^{2 / 3} f_{0}^{5 / 3}
$$

where $V_{R}$ is the rocket velocity and choosing a frequency $f_{0}$ in the range $4-60 \mathrm{~Hz}$. Since the power spectrum $P(f)$ depends upon the frequency as $f^{-5 / 3}$ in the inertial subrange, we can then integrate the power spectrum over the frequency range $4-60 \mathrm{~Hz}$ described above to obtain $\Delta n / n$. From thereon we can use the previous equations (1)-(4) to derive $\epsilon$ in a consistent manner. In each case, the $\epsilon$ profiles were then corrected for negative ion presence as described by
Hall (1997a). The correction has negligible consequences if the turbulence was significant. This is because one effect of the negative ions is to introduce a narrow viscous-convective subrange, and this will contribute relatively little if the inertial subrange is large; the reader is referred to Hall (1997a) for estimates of the magnitudes of negative ion effects.

\subsection{Ionisation gauge (TOTAL/CONE)}

The TOTAL and CONE instruments have been discussed in the literature and we will therefore only give the most important information here. Both instruments are in principle ionisation gauges measuring the neutral density and neutral density fluctuations with high precision (instrumental noise less than $0.1 \%$ of the total signal) and resolution (better than a few metres). The basic difference between the two instruments is that TOTAL is closed while CONE is an open ionisation gauge. The latter is much less influenced by the flow round the instrument and is therefore not as sensitive to ram (aerodynamic sense) effects. The neutral density fluctuations have been used to derive energy dissipation rates following the method outlined by Lübken (1996) and Lübken et al. (1993) and will not be repeated here. The basic principle of the method is to use the power spectrum of the observed density fluctuations and a theoretical model to obtain the best fit to the spectrum (see Lübken et al. (1993) for further details). From this fit, the inner scale $l_{0}$ indicating the transfer from the inertial to viscous subranges in the spectrum and hence the energy dissipation rate $\epsilon$ is obtained. Although this method does not directly estimate the kinetic energy spectrum, the matter of partition of energy is nonetheless implicit in the theoretical spectrum to which the data are fitted. The estimation of $\epsilon$ depends on the relation between $\eta$, the Kolmogorov microscale and $l_{0}$, the inner scale, and this is often given by $l_{0}=C_{4} \eta$. Lübken et al. (1993) show how $C_{4}$ may be 9.9 using the formulation of Heisenberg (1948), or 7.06 using that of Tartarskii (1971). Specifically, for the former:

$$
C_{4}=l_{0} / \eta=2 \pi\left\{\frac{9 f_{\alpha} a^{2} \Gamma(5 / 3) \sin (\pi / 3)}{16 \operatorname{Pr}_{\mathrm{n}}^{\mathrm{mol}}}\right\}^{3 / 4} .
$$

Here $\operatorname{Pr}_{\mathrm{n}}^{\mathrm{mol}}$ is the molecular Prandtl number; $f_{\alpha}$ is a factor equal to either 1 or 2 accounting for different nomalisations of the rate at which inhomogeneity is removed by molecular diffusion; $a^{2}$ is an experimentally determined constant (which we shall discuss later). It is from the spectral determination of $l_{0}$ combined with Eq. (7) and

$$
\eta=\left(\frac{v^{3}}{\epsilon}\right)^{1 / 4}
$$

$v$ being the kinematic viscosity (a function of both density and temperature), that we may determine $\epsilon$.

\subsection{Nose tip probe (NTP)}

The nose tip probe measuring electron density fluctuations has been discussed by, for example, Ulwick et al. (1988). The probe consists of an isolated tip (about $4 \mathrm{~cm}$ in length) of the nose cone of the rocket and is held at a fixed potential of $+3 \mathrm{~V}$ with respect to the rocket skin. The data were sampled at $8000 \mathrm{~Hz}$ giving an effective height resolution of better than $1 \mathrm{~m}$. Energy dissipation rates $\epsilon$ were derived using the so-called $C_{\mathrm{n}}^{2}$-method described above and for example by 
Blix et al. (1990) using spectra of electron density fluctuations as input. The data employed here have previously been published by Kelley et al. (1990).

\section{MF Radar Signal Fading Time Method}

We may illuminate the mesosphere and lower thermosphere using an MF radar. Structures in the electron density, at heights and times where the atmosphere is ionised to some degree, may give rise to sharp gradients in the refractive index at the transmitted frequency. These gradients scatter radiation (by either partial reflection or volume scatter) to form an interference pattern on the ground. Spaced antennae detect the movement of this pattern (moving with twice the speed of the scatterers), giving indications of the horizontal wind. In addition, however, the signals fade due to the scatterer motion (both uniform and fluctuating) and dissipation of the scatterers. The fluctuating component of the motion is used to estimate turbulent strength: the characteristic fading time may be related to the eddy diffusivity and hence the turbulent energy dissipation rate. This kind of measurement is described by Hocking (1997) who describe other methods, and compare with this one. The details of the method of correlating the signals from each of the spaced receivers are addressed by Briggs (1984), and the method is commonly referred to as the Full Correlation Analysis (FCA).

Descriptions of the experimental set up of the joint University of Troms $\varnothing /$ University of Saskatchewan MF radar may be found via Hall et al. (1998). The signal fading times, $\tau_{c}$, and velocity fluctuations, $v^{\prime}$, appropriate to an observer moving with the background wind, are computed according to Briggs (1984). In particular, and as used by Hall et al. (1998), at a height resolution of $3 \mathrm{~km}$ and time resolution of 5 minutes we use:

$$
v^{\prime}=\frac{\lambda \sqrt{\ln 2}}{4 \pi \tau_{c}}
$$

where $\lambda$ is the radar wavelength. The energy dissipation rate may be arrived at by dividing the kinetic energy of the turbulence, related to $v^{\prime 2}$, by a timescale (Blamont, 1963). In previous studies, (e.g., Manson et al., 1981) the BruntVäisälä period has been identified as a suitable timescale, the fluctuation as the vertical component, $w$, and the expression $\epsilon=0.4 w^{2} \omega_{\mathrm{B}}$ has been used ( $\omega_{\mathrm{B}}$ being the Brunt-Väisälä frequency). If we derived the velocity variance by a line-ofsight Doppler measurement the expression would be different as described exactly by Hocking (1983). Here, however, we have assumed that all three components of the velocity fluctuation are responsible for the fading of the signal and have thus chosen to use

$$
\epsilon^{\prime}=0.8 v^{\prime 2} / T_{\mathrm{B}}
$$

(Hall et al., 1998) where $T_{\mathrm{B}}$ is the Brunt-Väisälä period in seconds. Note that we recognise that the spatial scale inherent in the experiment cannot preclude buoyancy-scale fluctuations, because the outer scale of turbulence, $L_{\mathrm{B}}$ can be expected to be as little as $200 \mathrm{~m}$ in the mesosphere. There is a very real danger of gravity wave contamination that would lead to overestimates of $\epsilon$, the consequences for both spectral-width and velocity-fluctuation based methods being addressed by Hocking (1996). We therefore choose to introduce $\epsilon^{\prime}$ to distinguish our estimate from $\epsilon$. To use a formulation for $\epsilon$ which assumes a radar completely free from beam broadening here would have been clearly inappropriate. We have therefore attempted to address the warnings of Hocking (1983) (somewhat weakened by Vandepeer and Hocking (1993)) by accepting that $v^{\prime}$ is an estimate of total velocity perturbations within the scattering volume and choosing a time scale accordingly. We have attempted to justify this philosophy by checking for correlation between the estimates of $\epsilon^{\prime}$ and the total wind amplitude: a clear correlation might be anticipated if beam-broadening was significantly enhancing our estimate, which was not the case (Hall et al., 1998).

\section{EISCAT-Derived Characteristic Vertical Wave- number Method}

The EISCAT (Baron, 1984) radar at Ramfjordmoen $\left(69^{\circ} \mathrm{N}\right.$, $19^{\circ} \mathrm{E}$ ) in Northern Scandinavia is periodically run in a mode optimised for the mesosphere (Collis and Röttger, 1990). Data from this mode includes vertical soundings of the height range $70-90 \mathrm{~km}$ and results are made available which provide estimates of the vertical component of the neutral air motion (Collis, 1987). The time resolution is 5 minutes and the height resolution $1.05 \mathrm{~km}$. The data incorporated in this study are summarised in Table 4.

A typical EISCAT dataset giving vertical velocities over a total interval of $1 \frac{1}{2}$ days might exhibit broad regions of alternate upward and downward motion due to tides, semiand terdiurnal being common modes; these are invariably modulated by the shorter period motions of gravity wavessometimes showing very clearly, but often displaying a quasichaotic nature due to superposition of many periodicities. Furthermore, operations may be interrupted by transmitter and other failures. Low signal-to-noise ratios, particularly during geomagnetically quiet conditions at night and at low altitudes, impair extraction of Doppler shifts from the incoherent scatter spectra. We shall see how consequences of these problems are minimised in the vertical wavenumber method that follows. The consequences are direr for the velocity fluctuation method outlined in Section 5.

In order to tolerate intermittent data, spectral analyses are performed on the time-height data arrays using the LombScargle method described by Press and Rybicki (1989). This method is particularly useful for our purposes because not only is it designed for irregularly spaced data, but it yields both a characteristic frequency (the wavenumber, $m *$, in our case) and the significance that this is a true periodicity and not noise. For each timestep, we determine the periodogram including all available dynamics scales and then its characteristic wavenumber and significance. These spectra and their characteristic wavenumbers exhibiting a 95\% confidence are averaged together. The average characteristic wavenumber, which represents gravity waves, is taken to be equivalent to the $m *$ as defined in Fritts and van Zandt (1993). An obvious disadvantage in our strategy is that we are only obtaining an average for the height range 70-90 km (indeed, the data between 70 and $75 \mathrm{~km}$ tends to be sparse). According to Fritts and van Zandt (1993) this determination of $\epsilon$ should in fact be regarded as an upper limit (Fritts, private communication) in the same way as for the MF method just described. 
Table 4. Dates of EISCAT experiments and numbers of $95 \%$ confidence $m *$ samples as used in this study. Also included are the average $m *$ values ( $\mathrm{m}^{-1}$ ) and vertical velocity variances $\left(\mathrm{m}^{-2} \mathrm{~s}^{-2}\right)$ for each period.

\begin{tabular}{lrrrc}
\hline \multicolumn{1}{c}{ Date } & & No. of samples & $m *\left(\mathrm{~m}^{-1}\right)$ & Variance $\left(\mathrm{m}^{-2} \mathrm{~s}^{-2}\right)$ \\
\hline 12 June & 1990 & 116 & $6.16 \mathrm{E}-05$ & 2.76223 \\
30 July & 1990 & 97 & $5.53 \mathrm{E}-05$ & 3.98875 \\
27 August & 1990 & 5 & $6.52 \mathrm{E}-05$ & 1.4654 \\
12 February & 1991 & 4 & $5.33 \mathrm{E}-05$ & 3.0578 \\
20 February & 1991 & 2 & $5.19 \mathrm{E}-05$ & 5.74342 \\
17 March & 1991 & 55 & $5.97 \mathrm{E}-05$ & 1.50099 \\
4 June & 1991 & 149 & $5.68 \mathrm{E}-05$ & 1.74011 \\
10 July & 1991 & 84 & $5.87 \mathrm{E}-05$ & 3.48824 \\
10 June & 1992 & 7 & $5.57 \mathrm{E}-05$ & 6.21632 \\
30 July & 1992 & 299 & $5.79 \mathrm{E}-05$ & 4.37461 \\
27 October & 1992 & 25 & $6.22 \mathrm{E}-05$ & 2.08728 \\
20 January & 1993 & 33 & $5.14 \mathrm{E}-05$ & 7.37154 \\
15 June & 1993 & 49 & $5.65 \mathrm{E}-05$ & 2.90204 \\
20 July & 1993 & 7 & $5.37 \mathrm{E}-05$ & 6.28883 \\
14 September & 1993 & 14 & $6.58 \mathrm{E}-05$ & 0.654379 \\
15 March & 1994 & 68 & $6.23 \mathrm{E}-05$ & 1.90955 \\
11 August & 1994 & 27 & $5.51 \mathrm{E}-05$ & 3.05667 \\
2 May & 1995 & 322 & $6.66 \mathrm{E}-05$ & 1.93373 \\
17 December & 1996 & 30 & $4.89 \mathrm{E}-05$ & 5.92427 \\
\hline
\end{tabular}

The advantage of this method is its robustness against uncertainties in vertical velocity for two reasons: (a) we only use significant periodicities, excluding random variations due to instrument noise, and (b) a white noise background would not be expected to change the characteristic vertical wavelength appreciably.

Given an estimate of $m *$, Fritts and van Zandt (1993) then give the total gravity wave energy as:

$$
E_{0}=\frac{\omega_{\mathrm{B}}^{2}}{10 m_{*}^{2}}
$$

wherein we use the value of the Brunt-Väisälä frequency, $\omega_{\mathrm{B}}$, given by Fritts and van Zandt (1993) for consistency. Similarly, and with reference again to Fritts and van Zandt, we define the energy scale height $H_{\mathrm{E}}=2.3 H(H=6.3 \mathrm{~km})$ and so finally:

$$
\epsilon^{\prime} \approx \frac{\omega_{\mathrm{B}} E_{0}}{18 m_{*}}\left(\frac{1}{H}-\frac{3}{2 H_{\mathrm{E}}}\right)
$$

hence we obtain estimates for an upper limit of energy dissipation, which we again denote by $\epsilon^{\prime}$. From the above equations, we can see that a $10 \%$ change in $\omega_{\mathrm{B}}$ would correspond to a $30 \%$ change in $\epsilon^{\prime}$. The sensitivity to $H_{\mathrm{E}}$ is less, a $10 \%$ change here corresponding to a $10 \%$ change in $\epsilon^{\prime}$. These are acceptable if one notes that considerable notoriety in estimates of $\epsilon^{\prime}$ stem from order of magnitude disagreements between instruments, methods and interpretations.

\section{EISCAT-Derived Vertical Velocity Fluctuation Method}

The experiment description from the previous section applies also to this method. Here a very simplistic approach is used by Hall (1997b) that avoids entailing any model atmosphere. The assumptions are simple to comprehend, but at the same time raise the question as to viability. We simply take adjacent determinations of the vertical velocity (recall the EISCAT height resolution for the experiment in question is $1.05 \mathrm{~km}$ ) and assume the difference to be the velocity fluctuation corresponding to an approximately $1 \mathrm{~km}$ eddy. Clearly, if the outer scale is only $200 \mathrm{~m}$, for example, it is unreasonable to talk of turbulence, and so Hall (1997b) refers to the resulting entity as a kilometer-scale kinetic energy fluctuation, $\epsilon_{1 \mathrm{~km}}$ :

$$
\epsilon_{1 \mathrm{~km}}(z)=[\overline{w(z, t)-w(z+1050, t)}]^{3} / 1050
$$

where $w(z, t)$ is the vertical velocity at height $z$ and time $t$. Here we have introduced yet another kind of energy dissipation rate, which one should think of as an overestimate due to an imposed length scale. In the other ground-based methods described here, an upper limit is calculated with premeditated use of assumptions. The velocity fluctuation method was inspired by the work of Blamont (1963) in which such fluctuations and their approximations to various definitions of structure function are discussed in depth. Blamont (1963) 


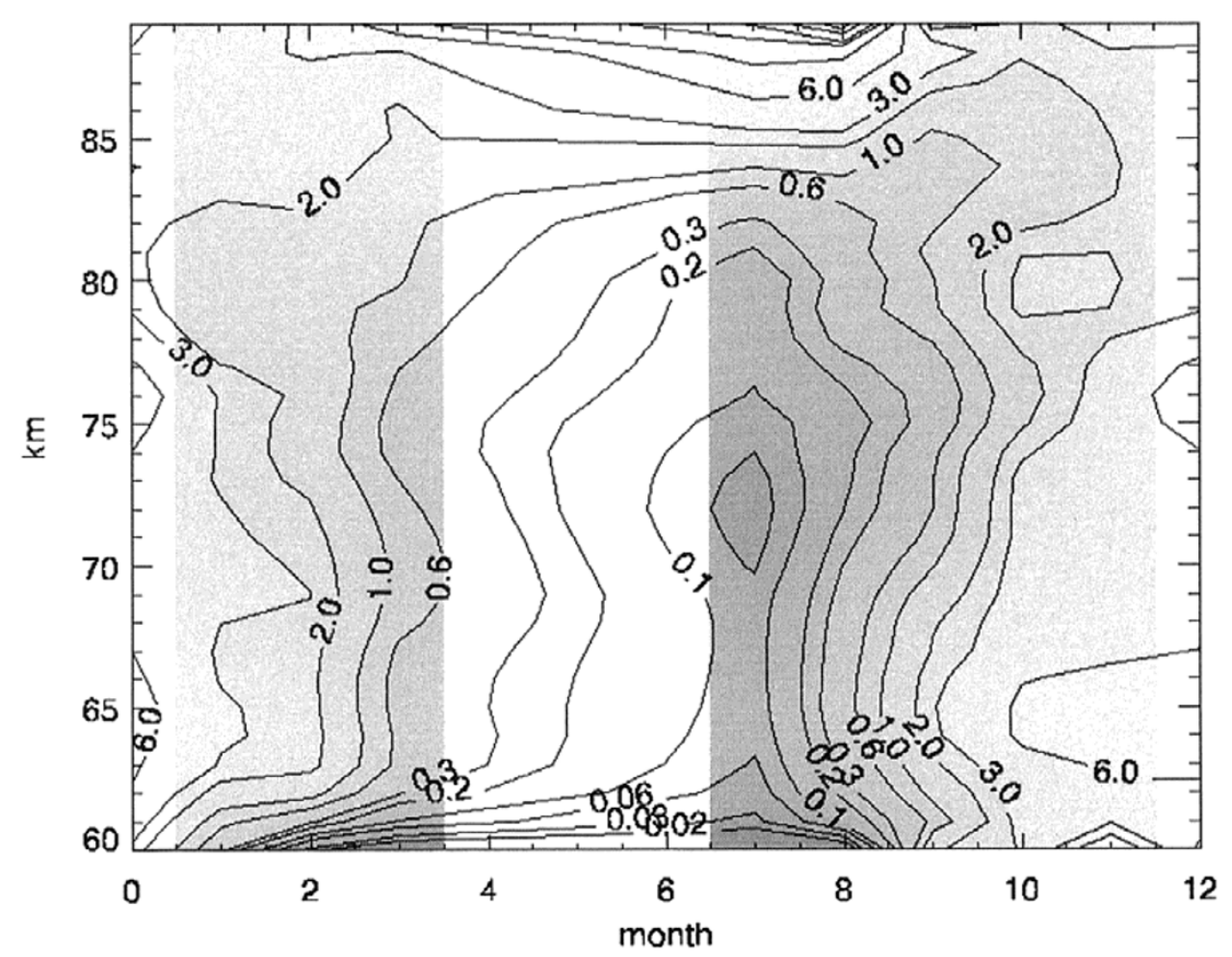

Fig. 2. In situ results. The soundings listed in Table 2 have been analysed using the methods described in the text. From Table 3 we note that no data was available for April, May, June or December; the December profile is an average of the November and January profiles; the spring/early summer data is replaced by the kinematic viscosity multiplied by the square of the Brunt-Väisälä frequency, this representing a minimum value of $\epsilon$ (e.g., Lübken et al., 1993). Units on the contours are in $\mathrm{mWkg}^{-1}$. Months with missing data are denoted by lighter shading.

furthermore illustrates an excellent description of the theory by application to sodium cloud observations.

\section{Results}

All the methods described above yield profiles of " $\epsilon$ " as functions of season, but with the exception of the characteristic wavenumber approach. Figures $2-4$ present results from each of the first three methods. Note that the scales differ somewhat, but to allow for this, the contour levels have been labelled.

For the in situ results for the months of April, May and June, the expression $\epsilon_{\min }=v \cdot \omega_{\mathrm{B}}^{2}$ has been used, $v$ being the kinematic viscosity, (e.g., Lübken et al., 1993) (cf. Eq. (14)). December, representing only a one-month gap, is the average of January and November. Subsequently a $3 \times 3$ point boxcar smoothing has been applied to the original resolutions of 1 month and $1 \mathrm{~km}$ (Fig. 2).

The MF fading time method yields daily profiles each with a $3 \mathrm{~km}$ height resolution. We show the data for 1997 here in Fig. 3. Due to transmitter problems, January data has been omitted. A one-week boxcar smoothing was applied.

The characteristic vertical wavenumber method has provided values of $\epsilon^{\prime}$ each of which is an average of typically 1-2 days. The points are plotted according to date, ignoring the year, and no smoothing has been used (Fig. 4). As we see, the characteristic wavenumber method yields a simple time series; each value is derived from a profile of vertical velocities, but not all measurement heights yield reliable data. We find that the average height (each $m *$ is associated with a representative height depending on the useable velocity values, and these representative heights may then be averaged) is around $79-80 \mathrm{~km}$.

The vertical velocity fluctuation method yields profiles at each of the dates the characteristic vertical wavenumber method did. Since we feel that this method was rather an exploratory foray into the use of EISCAT to investigate turbulence we prefer not to present such results explicitly here.

Let us now review the salient points of these figures:

i. Estimates of $\epsilon$ from in situ measurements (Fig. 2) reveal an annual variation below $85 \mathrm{~km}$ with almost no turbulence in summer and almost constant turbulence with height in winter. Above $85 \mathrm{~km}$ there is weak evidence for a semi-annual variation, although the clear feature is the presence of strong turbulence at $89 \mathrm{~km}$ in late summer.

ii. The MF results (Fig. 3) show relatively low turbulence levels in the spring and summer, and a more even distribution of turbulence with height in winter. Thus, below $80 \mathrm{~km}$ there is an annual variation. Above $80 \mathrm{~km} \mathrm{a}$ semi-annual oscillation (SAO) signature is quite obvious. Minima at the equinoxes have also been reported by Hocking $(1988,1991)$.

iii. The characteristic vertical wavenumber method (Fig. 4) exhibits a clear SAO signature again similar to the findings reported by Hocking $(1988,1991)$. 


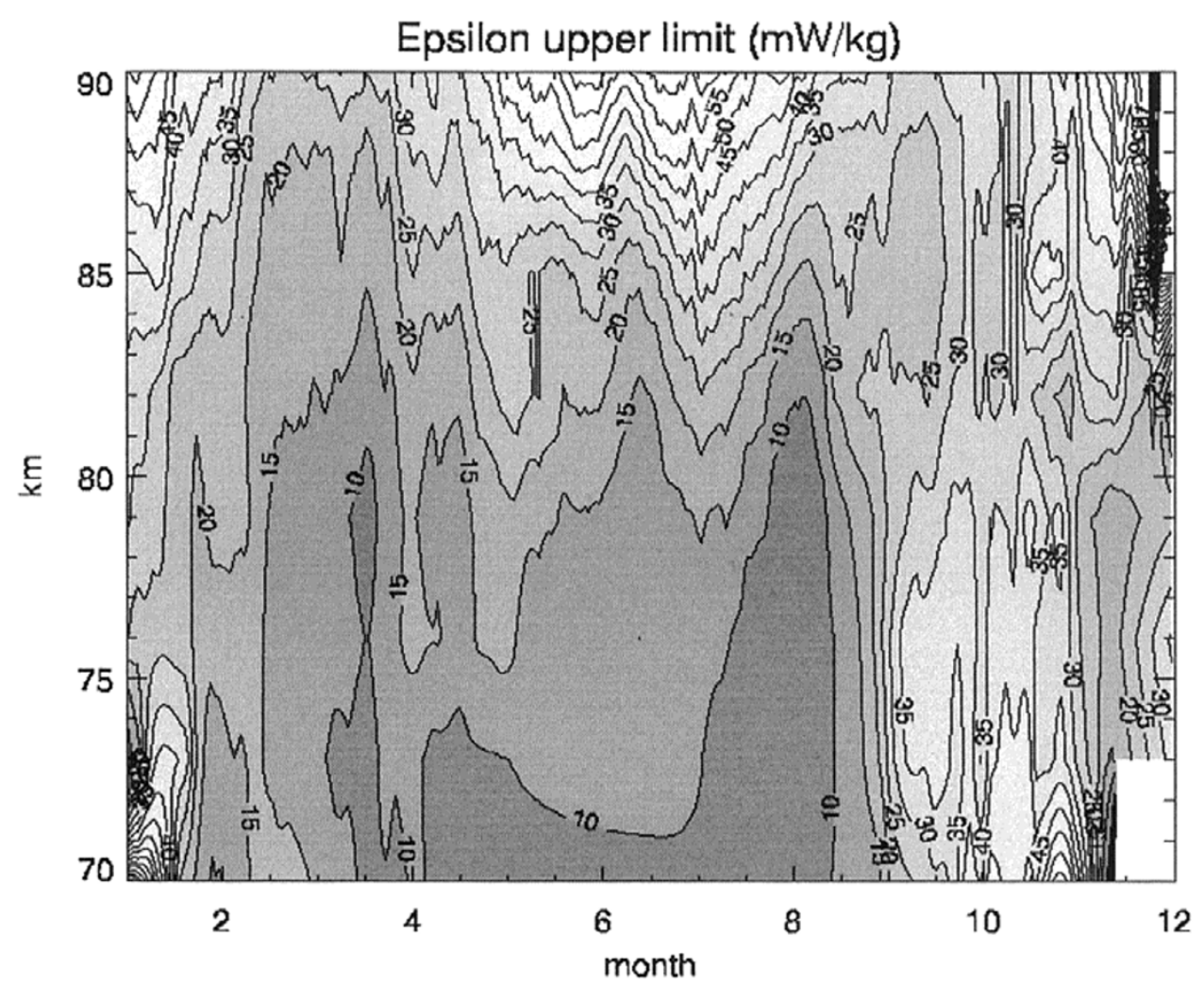

Fig. 3. MF fading time results. Again units of the contour labels are $\mathrm{mWkg}^{-1}$

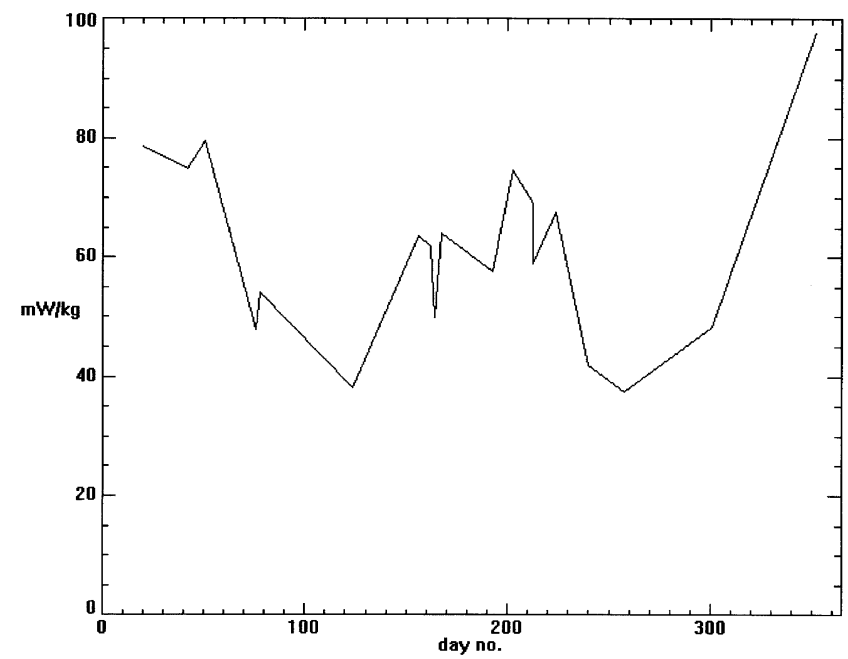

Fig. 4. EISCAT characteristic wavenumber results. See text regarding which height is represented.

\section{Discussion}

Let us compare the results presented in the previous section. Figure 5 presents data from all 4 methods for $90 \mathrm{~km}$. In the case of in situ methods, we have included variance bars obtained when averaging the individual profiles, the numbers of samples having been indicated in Table 3 . The most noteworthy features in Fig. 5. are:

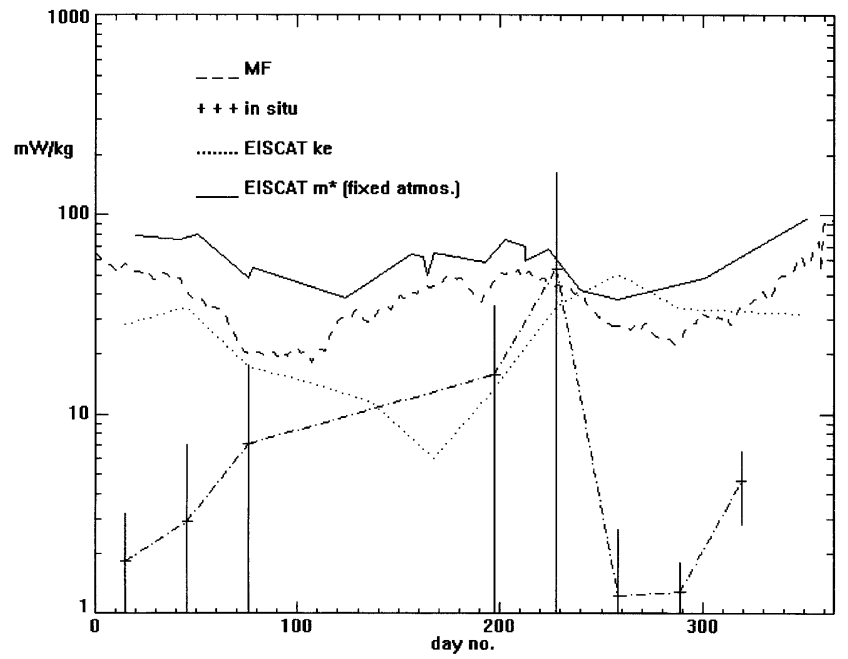

Fig. 5. Comparison of: in situ results for $88 \mathrm{~km}$, MF results for $88 \mathrm{~km}$, EISCAT characteristic wavenumber (labelled "EISCAT $m *$ "), EISCAT velocity fluctuation for $88 \mathrm{~km}$ (labelled "EISCAT ke"). Again units are $\mathrm{mWkg}^{-1}$. Standard deviations obtained from the averaging of the individual in situ soundings are indicated as vertical bars.

i. The EISCAT characteristic wavenumber and MF fading time methods for $\epsilon$ upper limits agree well, both in absolute value and SAO behaviour.

ii. The EISCAT vertical velocity fluctuation method agrees well with the other ground-based methods except during 
summer, remarkable considering the naive nature of the method. This method fails to indicate an SAO.

iii. The in situ methods collectively exhibit systematically lower turbulent intensities. The SAO is not convincingly present. Only in summer do the intensities approach those suggested by the other methods. It would be surprising if these methods agreed in magnitude with the radar methods in (i), however, since the in situ measurements give estimates of $\epsilon$ as opposed to upper limits for $\epsilon$.

Similar SAO signatures in $\epsilon$ have been reported for midlatitudes: Hocking $(1988,1991)$. Due to the almost inevitable gravity wave contamination, the absolute values in the MF results should be reduced by a factor 2 following recommendations found in Hocking (1996). This reduction probably applies to the EISCAT results also, but not the in situ methods.

The differences in findings from the various estimates of turbulent intensity can have many causes. What comes to the fore here, however, is that it is not always the absolute intensities that disagree, but also the seasonal variation. We must recall that the EISCAT characteristic wavenumber and MF fading time methods indicate upper limits, so that the real $\epsilon$ must be less than the minimum of the values indicated by the two methods. At the same time, we require that other methods indicate values of $\epsilon$ between the minimum energy dissipation supported by the atmosphere, $\epsilon_{\min }$, defined by

$$
\epsilon_{\min }=v \omega_{\mathrm{B}}^{2} / \beta
$$

where $\beta$ is a constant, $\nu$ is the kinematic viscosity and $\omega_{\mathrm{B}}$ is the Brunt-Väisälä frequency (Fukao et al., (1994) use a value of 0.3 for $\beta$ ), and the upper limit identified by the radar methods. We see from Fig. 5 that this is indeed the case.

Several authors have addressed the differences between the absolute values: Blix (1993) focussed on in situ problems, whereas Hocking (1999) gives a most up-to-date review independent of method. For mid-latitudes, Manson and Meek (1987) used MF-radar derived winds to investigate the seasonal variation of gravity wave intensity. These authors derived both energy dissipation rates and eddy diffusivities from both the gravity wave intensities and the signal fading times (quite independent approaches); agreement was good and a strong SAO was observed both in ground based data and in situ determined wind-shear.

When addressing discrepancies, one is tempted to examine the applicability of the numerous constants, examined exhaustively by Hocking (1999). We must note, however, that adjustments to the majority of these constants will only lead to systematic increases or decreases in the levels of the individual time series. Although different constants are required for different methods, such adjustments do have the potential to draw the methods more into line (or make the agreement worse!). These kinds of adjustments will only lead to modification of the apparent seasonal variation if the methods themselves varied as time of year (e.g., the $C_{n}^{2}$ method was used to analyse winter soundings and the spectral fit method for summer soundings). This is not the case, at least, not to such a degree as to introduce an SAO in the in situ time series, and so we should look elsewhere.
The object of this study has been to summarise some contemporary studies of seasonal variation of turbulent intensity from recently published material. In addition, we outline the methods used to analyse the original data, be it plasma or neutral density fluctuations or estimates of turbulent velocity, and thus highlight the underlying assumptions. In order to comprehend the physics underlying these assumptions, the reader is encouraged to refer to Hocking (1999) to which this review is intended as a companion paper.

In order to illustrate the problems involved, however, consider the method of fitting a theoretical spectrum to directly measured neutral density fluctuations. This method attempts to determine $\epsilon$ rather than an upper limit and furthermore, by measuring properties of the neutral gas itself avoids the problem of deriving neutral density fluctuations from plasma density fluctuations. It would appear that (for this method) the choice of the constant $C_{4}$ is the major point open to discussion. Apart from a dependency on the molecular Prandtl number, the expressions for $C_{4}$ from both Heisenberg (1948) and Tartarskii (1971) involve the factor $a^{2}$ which Lübken et al. (1993) take from Hill (1978). Referring to Hocking (1999), however, we find that $a^{2}$ may exhibit a Richardson Number dependence. While taking $R_{\mathrm{i}}=0.4$ as a typical "maintained" turbulent atmosphere may be suitable for the kind of averaging used in the MF method, it may be difficult to defend a generalisation for use in individual in situ soundings prior to averaging. The final expression for Kolmogorov microscale in terms of $\epsilon$ furthermore entails an estimation of the kinematic viscosity, which is both density and temperature dependent. A seasonal variation in the degree of layering (and hence $R_{\mathrm{i}}$ ) is only to be expected from the well-known summer-winter differences in the temperature structure. The other methods are equally, if not even more, prone to these seasonal differences due to their use of models for scale height and buoyancy frequency. Even when other supporting soundings have provided information on density, wind and temperature structure, it may be hard to argue that these values are applicable to the density fluctuation sounding itself because there may easily be separation in space and/or time corresponding to gravity wave timescales. Thus any determinations of, for example $R_{\mathrm{i}}, v$ or $\omega_{\mathrm{B}}$ may be questionable, and all these parameters will be expected to exhibit seasonal variability.

Acknowledgments. We would like to acknowledge the staff of the EISCAT Scientific Association which is supported by the research councils of Finland, France, Germany, Japan, Norway, Sweden and the United Kingdom. Thanks go to Bjørnar Hansen and Bjørn-Ove Husøy of the Auroral Observatory in Troms $\varnothing$ for patient maintenance of the MF radar. This work would not have been possible without the help and results of Franz-Josef Lübken to whom the authors are particularly indebted. Finally we would like to thank Dr. Wayne Hocking and one anonymous referee for their invaluable input as reviewers of this manuscript.

\section{References}

Baron, M., The EISCAT facility, J. Atmos. Terr. Phys., 46, 469-472, 1984. Blamont, J.-E., Turbulence in atmospheric motions between 90 and $130 \mathrm{~km}$ of altitude, Planet. Space Sci., 10, 89-101, 1963.

Blix, T. A., In-situ studies of turbulence and mixing; problems and questions, in Coupling Processes in the Lower and Middle Atmosphere, edited by Thrane et al., pp. 329-344, Kluwer, Dordrecht, The Netherlands, 1993. 
Blix, T. A., E. V. Thrane, and Ø. Andreassen, In Situ Measurements of the fine-scale structure and turbulence in the mesosphere and lower thermosphere by means of electrostatic ion probes, J. Geophys. Res., 95(D5), 5533-5548, 1990.

Briggs, B. H., The analysis of spaced sensor records by correlation techniques, Handb. MAP, 13, 166-186, 1984.

Collis, P. N., Common program data analysis at EISCAT, in Proceedings of the EISCAT Annual Review Meeting, Skibotn, Norway 2-5 March, 1987, edited by P. N. Collis, 1987.

Collis, P. N. and J. Röttger, Mesospheric studies using the EISCAT UHF and VHF radars: a review of principles and experimental results, J. Atmos. Terr. Phys., 52, 569-584, 1990.

Fritts, D. C. and T. E. van Zandt, Spectral estimates of gravity wave energy and momentum fluxes, J. Atmos. Sci., 50, 3685-3694, 1993.

Fukao, S., M. D. Yamanaka, N. Ao, W. K. Hocking, T. Sato, M. Yamamoto, T. Nakamura, T. Tsuda, and S. Kato, Seasonal variability of vertical eddy diffusivity in the middle atmosphere, 1 . Three-year observations by the middle and upper atmosphere radar, J. Geophys. Res., 99, 18,973-18,987, 1994.

Hall, C. M., The influence of negative ions on mesospheric turbulence traced by ionisation: implications for radar and in situ experiments, J. Geophys. Res., 102, 439-443, 1997a.

Hall, C. M., Kilometer scale kinetic energy perturbations in the mesosphere derived from EISCAT velocity data, Radio Sci., 32, 93-101, 1997b.

Hall, C. M., C. E. Meek, and A. H. Manson, Turbulent energy dissipation rates from the University of Troms $\varnothing /$ University of Saskachewan MF radar, J. Atmos. Sol.-Terr. Phys., 60, 437-440, 1998.

Hedin, A. E., Extension of the MSIS thermosphere model into the middle and lower atmosphere, J. Geophys. Res., 96, 1159-1172, 1991.

Heisenberg, W., On the theory of statistical and isotropic turbulence, Proc. R. Soc. London, 195, 402-406, 1948.

Hill, R. J., Models of the scalar spectrum for turbulent advection, J. Fluid. Mech., 88, 541-562, 1978.

Hillert, W., F.-J. Lübken, and G. Lehmacher, Neutral air turbulence during DYANA as measured by the TOTAL instrument, J. Atmos. Terr. Phys., 56, 1835-1852, 1993.

Hocking, W. K., On the extraction of atmospheric turbulence parameters from radar backscatter Doppler spectra-I. Theory, J. Atmos. Terr. Phys., 45, 89-102, 1983.

Hocking, W. K., Measurement of turbulent energy dissipation rates in the middle atmosphere by radar techniques: A review, Radio Sci., 20, 14031422, 1985.

Hocking, W. K., Two years of continuous measurements of turbulence parameters in the upper mesosphere and lower thermosphere made with a 2-MHz radar, J. Geophys. Res., 93, 2475-2491, 1988.

Hocking, W. K., The effects of middle atmosphere turbulence on coupling between atmospheric regions, J. Geomag. Geoelectr., 43, Suppl., 621636, 1991.

Hocking, W. K., On the relationship between the strength of atmospheric radar backscatter and the intensity of atmospheric turbulence, $A d v$. Space Res., 12, (10)207-(10)213, 1992.

Hocking, W. K., An assessment of the capabilities and limitations of radars in measurements of upper atmosphere turbulence, Adv. Space Res., 17, (11)37-(11)47, 1996.

Hocking, W. K., Strengths and limitations of MST radar measurements of middle-atmosphere winds, Ann. Geophysicae, 15, 1111-1122, 1997.

Hocking, W. K., The dynamical parameters of turbulence theory as they apply to middle atmosphere studies, Earth Planets Space, 51, this issue 525-541, 1999.

Hocking, W. K. and P. K. L. Mu, Upper and middle tropospheric kinetic energy dissipation rates from measurements of $\overline{C_{\mathrm{n}}^{2}}$-review of theories, in situ investigations, and experimental studies using the Buckland Park atmospheric radar in Australia, J. Atmos. Sol.-Terr. Phys., 59, 1779-1803, 1997.

Hoppe, U.-P., T. Eriksen, E. V. Thrane, T. A. Blix, J. Fiedler, and F.-J. Lübken, Observations in the polar middle atmosphere by rocket-borne Rayleigh lidar: First results, Earth Planets Space, 51, this issue, 815824, 1999.

Kelley, M. C., J. C. Ulwick, J. Röttger, B. Inhester, T. Hall, and T. Blix, Intense turbulence in the polar mesosphere: rocket and radar measurements, J. Atmos. Terr. Phys., 52, 875-892, 1990.

Kolmogorov, A. N., The local structure of turbulence in incompressible viscous fluids for very large Reynolds numbers, C. R. Acad. Sci. URSS, 30, 301-305, 1941.

Lübken, F.-J., Rocket-borne measurements of small scale structures and turbulence in the upper atmosphere, Adv. Space Res., 17, (11)25-(11)35 1996.

Lübken, F.-J., W. Hillert, G. Lehmacher, and U. von Zahn, Experiments revealing small impact of turbulence on the energy budget of the mesosphere and lower thermosphere, J. Geophys. Res., 98(D11), 20,369-20,384, 1993.

Manson, A. H. and C. E. Meek, Small-scale features in the middle atmosphere wind field at Saskatoon, Canada $\left(52^{\circ} \mathrm{N}, 107^{\circ} \mathrm{W}\right)$ : An analysis of MF radar data with rocket comparisons, J. Atmos. Sci., 44, 3661-3672, 1987.

Manson, A. H., C. E. Meek, and J. B. Gregory, Gravity waves of short period (5-90 $\mathrm{min}$ ) in the lower thermosphere at $52^{\circ} \mathrm{N}$ (Saskatoon, Canada): 1978/1979, J. Atmos. Terr. Phys., 43, 35-44, 1981.

Press, W. H. and G. B. Rybicki, Fast algorithm for spectral analysis of unevenly sampled data, Astrophys. J., 338, 277-280, 1989.

Tartarskii, V. I., The Effects of the Turbulent Atmosphere on Wave Propagation, Israel Programme for Scientific Translations, Jerusalem, 1971.

Thrane, E. V. and B. Grandal, Observations of fine scale structure in the mesosphere and lower thermosphere, J. Atmos. Terr. Phys., 43, 179-189, 1981.

Thrane, E. V., Ø. Andreassen, T. Blix, B. Grandal, A. Brekke, C. R. Philbrick, F. J. Schmidlin, H. U. Widdel, U. von Zahn, and F.-J. Lübken, Neutral air turbulence in the upper atmosphere observed during the Energy Budget Campaign, J. Atmos. Terr. Phys., 47, 243-264, 1985.

Ulwick, J. C., K. D. Baker, M. C. Kelley, B. B. Balsley, and W. L. Ecklund, Comparison of simultaneous MST radar and electron density probe measurements during STATE, J. Geophys. Res., 93, 6989-7000, 1988

Vandepeer, B. G. W. and W. K. Hocking, A comparison of Doppler and spaced antenna radar techniques for the measurement of turbulent energy dissipation rates, Geophys. Res. Lett., 20, 17-20, 1993.

Weinstock, J., Vertical turbulent diffusion in a stably stratified fluid, J. Atmos. Sci., 35, 1022-1027, 1978.

C. M. Hall (e-mail: chris.hall@phys.uit.no), U.-P. Hoppe, T. A. Blix, E. V. Thrane, A. H. Manson, and C. E. Meek 